data have been deposited in GenBank.

A draft sequence of the rice genome by the agricultural biotechnology company Monsanto, based in St Louis, Missouri, and one by Celera of the mouse genome, are also under preparation, but have not yet been scheduled for publication in any journal.

Syngenta currently makes its data available to a handful of academic groups through special agreements. The publication of Syngenta's rice genome in Science might result in changes to the company's policy, giving more researchers access to the sequence data. But, as the letter demonstrates, researchers remain deeply divided over the terms of such access. "This goes to the heart of what science is all about, the free exchange of ideas, data and reagents," says Bruce Stillman, director of the Cold Spring Harbor Laboratory in New York state. Science should not compromise on making the data freely available, he says.

But Ron Cantrell, director of the International Rice Research Institute in the Philippines, is more supportive of Science's decision to publish. "You have to ask the question 'is it better not to have any access at all?', ' he says, adding that, in his experience, Syngenta and Monsanto have "been very forthcoming" in collaborations with the public sector.

Chris Novak, a spokesman for Syngenta, says that the company hopes to work with the publicly funded International Rice Genome Sequencing Project (IRGSP). The project intends to produce a 'finished' highquality sequence, as opposed to the drafts, containing many gaps, that are about to be published.

Researchers point out that Science's agreement with Syngenta is not entirely analogous to the one it reached last year with Celera on the human genome. Celera contributed no data to the public Human Genome Project, instead relying on data from the public project to complete its own sequence. In contrast, Syngenta has already contributed significant mapping data to the IRGSP, through a collaboration with Clemson University in South Carolina.

But Syngenta has so far refused to share its raw sequence data with all of the public group - unlike its rival Monsanto, whose contributions of sequence data are credited with strongly accelerating the public project.

In January, however, Syngenta began talks with the IRGSP and, according to one IRGSP official, has agreed in principle to match the Monsanto agreement. If it does, "all the Syngenta and Monsanto data will be in the public domain by the end of the year", says the official. The likelihood of this happening might be a factor in persuading Science to accept restrictions on the rice data for the time being, observers suggest.

\title{
NASA urged to play waiting game on Hubble's retirement
}

\section{Tony Reichhardt, Washington}

Even as NASA completes a successful service mission on the Hubble Space Telescope, a debate is simmering in the agency about how long to keep the instrument in action.

NASA's current plan is to return the telescope to Earth in 2010 and put it in the Smithsonian National Air and Space Museum in Washington. But some Hubble-project scientists and engineers at NASA's Goddard Space Flight Center in Maryland have been arguing — albeit with little success so far - that the telescope should continue its observations.

The dilemma of when to pull the plug on a successful mission is a familiar one at NASA. But the case of Hubble will be especially difficult. Not only is it the most powerful telescope in history, but its observing grants are now established as a mainstay of support for US astronomers. And after last week's service mission - the fourth since its launch in 1990, and one which brought an infrared camera back to life and installed the powerful Advanced Camera for Surveys the telescope has never been more capable.

But NASA needs to retire Hubble to pay for its successor, the Next Generation Space Telescope (NGST). The agency has already delayed Hubble's retirement from 2005 to 2010, and is reluctant to do so again to appease those whom former NASA administrator Dan Goldin once derided as "Hubble huggers". So the current plan is for astronauts to install another camera and spectrograph during a fifth and final servicing mission in 2004, and to bring the telescope home six years later.

But project engineers at Goddard say that this will not be straightforward. Dismantling Hubble and returning it to Earth is more challenging than previously thought. The mission would require up to five spacewalks, and the telescope's weight would strain the shuttle's maximum landing limit.

NASA had hoped to launch the NGST

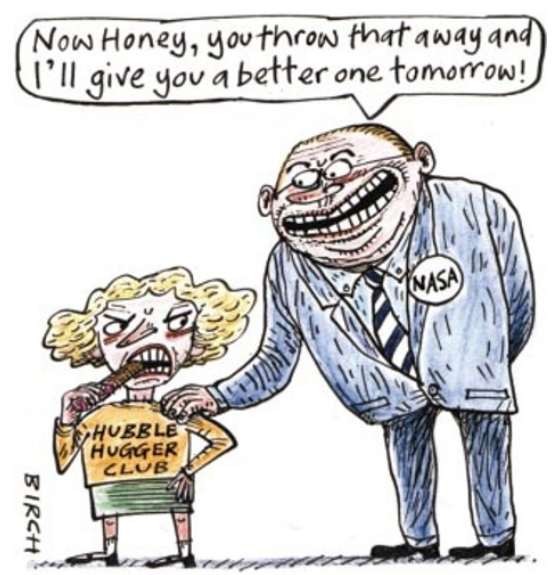

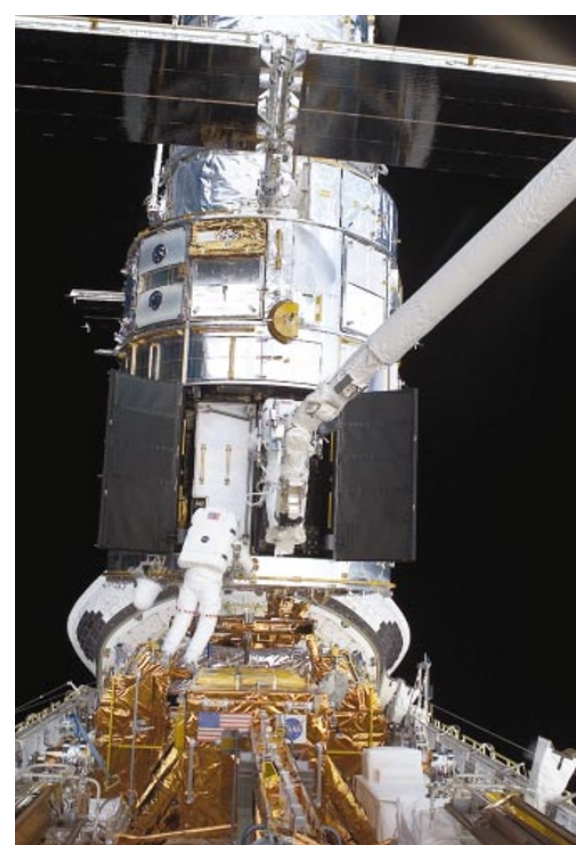

Despite the telescope's renewed capacity, NASA is reluctant to spend money on Hubble as it ages.

before Hubble retires, but the launch date has already moved from 2007 to 2009, and could easily slip further. According to Goddard engineers, Hubble is unlikely to survive six years after 2004 without maintenance.

So the Goddard team has proposed that another service mission be added in 2007. As well as making any necessary repairs, shuttle astronauts would attach a propulsion module that could allow the telescope to be 'deorbited' safely to burn up in the atmosphere.

The Air and Space Museum would lose an exhibit, but scientists would gain more Hubble viewing time. Hubble senior project scientist Dave Leckrone of Goddard, who advocates extending the telescope's life, admits it will be an uphill struggle to convince NASA. "The official position is that there will be no more servicing of Hubble after 2004," he says.

A committee representing Hubble users advised NASA last October to consider the extra servicing mission. Committee chairman George Miley of Leiden University in the Netherlands agrees that delaying the NGST would be "bad for astronomy", but adds that "the effectiveness of an extra Hubble refurbishment mission should be seen in a wider context and considered within the framework of the NASA programme as a whole".

But in a letter to Anne Kinney, director of NASA's 'Origins' programme, an advisory committee to that programme argued that "minimizing additional expenditures on [Hubble] is crucial to keep the development of NGST on track". 